\title{
Mechanical loading and control of breathing in patients with severe chronic obstructive pulmonary disease
}

\author{
R Duranti, G Misuri, M Gorini, P Goti, F Gigliotti, G Scano
}

\begin{abstract}
Background - High neural drive to the respiratory muscles and rapid and shallow breathing are frequently observed in patients with chronic obstructive pulmonary disease (COPD), and both mechanical and chemical factors are thought to play a part. However, the interrelation between these factors and the modifications in the control of breathing are not clearly defined. The effects of an acute decrease in mechanical load by the administration of a high dose of a $\beta_{2}$ agonist were studied.

Methods - Nine spontaneously breathing patients with severe COPD took part in the study. Criteria for entry were FEV $_{1}$ of $<40 \%$ of predicted and an improvement in $\mathrm{FEV}_{1}$ of $<200 \mathrm{ml}$ after inhalation of $400 \mu \mathrm{g}$ fenoterol. The following parameters were measured: lung volumes, tidal volume (VT), respiratory frequency (Rf), maximal pleural pressure during a sniff manoeuvre (PPLmax), pleural pressure swings (PPLsw), lung resistance (RL), RIJ Pplmax ratio, and surface electromyographic activity (EMG) of diaphragm (EDI) and parasternal (EPS) muscles. Arterial oxygen saturation $\left(\mathrm{SaO}_{2}\right)$, end tidal carbon dioxide pressure $\left(\mathrm{PETCO}_{2}\right)$, and the electrocardiogram were also monitored. Each variable was measured under control conditions and 20 and 40 minutes after the inhalation of $800 \mu \mathrm{g}$ fenoterol. In five patients the effects of placebo were also studied.
\end{abstract}

Results - Fenoterol resulted in an increase in $\mathrm{FEV}_{1}$ and decrease in FRC. $\mathrm{SaO}_{2}$ did not change, while $\mathrm{PETCO}_{2}$ fell and heart rate increased. The VT increased, and $R f$ decreased, Pplsw fell and PPLmax increased, thus the PPLsw/Pplmax ratio fell. Both $R \mathbf{L}$ and Ru/Pplmax also fell, and a substantial decrease in EDI and EPS was observed. Changes in PPLsw were related to changes in FEV $V_{1}$ and $R L$. Changes in VT and $R f$, and EDI/TI and EPS/TI were also related to changes in PPLsw and RI/PPLmax ratio, but not to changes in $F E V_{1}$. No variation was observed with placebo.

Conclusions - In patients with severe COPD a decrease in inspiratory muscle loading relative to the maximal available strength, as expressed by the RI/PPLmax and PPLsw/ Pplmax ratios, appears to be the major determinant of changes in breathing pattern and inspiratory muscle activity (decrease in EMG).

(Thorax 1995;50:127-133)

Keywords: chronic obstructive pulmonary disease, mechanical load, breathing pattern, respiratory muscles.

Patients with severe chronic obstructive pulmonary disease (COPD) have both a high neural drive to the respiratory muscles ${ }^{1-4}$ and a more rapid and more shallow pattern of breathing. ${ }^{4-6}$ Both mechanical ${ }^{16-8}$ and chemical ${ }^{19}$ factors are thought to be involved in determining these functions. Among the mechanical factors, an increase in airways resistance plays an important part in increasing neural inspiratory drive in both normal subjects ${ }^{1011}$ and in asthmatic patients. ${ }^{1112}$ On the other hand, the relationship between an acute increase in airways resistance and neural inspiratory drive appears to be more complex in patients with COPD. Altose et $a l^{7}$ reported that adding external inspiratory resistance produced no augmentation in mouth occlusion pressure $\left(\mathrm{P}_{0.1}\right)$. Furthermore, the $\mathrm{P}_{0.1}$ response seen with progressive hypercapnia did not occur during methacholine-induced bronchoconstriction. ${ }^{13}$ Pardy et $a l^{14}$ observed rapid shallow breathing with no change in mean inspiratory flow during histamine-induced airways resistance in patients with COPD, suggesting that an increase in airways resistance enhances central respiratory activity and modifies the pattern of breathing. An increase in airways resistance and a decrease in respiratory muscle strength may trigger the signal for the integrated response that controls the pattern of breathing. ${ }^{8}$ However, the interrelation of increased mechanical load relative to respiratory muscle strength and changes in neural inspiratory drive and breathing pattern has not yet been established.

We have investigated the effects of a large and rapid decrease in mechanical load following the administration of a large dose of a bronchodilator in a small group of patients with severe COPD.

\section{Methods}

SUBJECTS

Nine men with chronic obstructive pulmonary disease, as defined by the criteria of the Am- 
erican Thoracic Society, ${ }^{15}$ gave informed consent to the experimental procedures. Criteria for entry were: a forced expiratory volume in one second $\left(\mathrm{FEV}_{1}\right)$ of less than $40 \%$ of the predicted value; an improvement in $\mathrm{FEV}_{1}$ of less than $200 \mathrm{ml}$ after inhalation of $400 \mu \mathrm{g}$ fenoterol; a peripheral blood eosinophil count of less than $400 / \mathrm{mm}^{3}$; and no treatment with inhaled or oral corticosteroids. Inhaled bronchodilators were withdrawn 24 hours before the study.

\section{MEASUREMENTS}

Age, height and weight (expressed both as absolute value and as percentage predicted) were recorded. All respiratory measurements were performed with the patients seated. Routine spirometry was obtained by a water sealed spirometer (Godart); functional residual capacity (FRC) was measured by the helium dilution technique. Arterial blood gases were also measured with the subjects breathing room air. The normal values for lung volumes were those of the European Community for Coal and Steel. ${ }^{16}$

For ventilation measurements patients breathed through a Fleisch no. 3 pneumotachograph connected to a flow transducer. Volume was obtained from electrical integration of the flow signal. From the spirogram we derived: inspiratory time ( $\mathrm{T} I)$, expiratory time $(\mathrm{TE})$, total time of the respiratory cycle (Tтот), and tidal volume (VT). Mean inspiratory flow $(\mathrm{VT} / \mathrm{TI})$, duty cycle (TI/TTот), respiratory frequency $(\mathrm{R} f=1 /$ TTOT $\times 60)$, and instantaneous ventilation $(\mathrm{VE}=\mathrm{VT} \times \mathrm{R} f)$ were also calculated. End tidal carbon dioxide pressure $\left(\mathrm{PETCO}_{2}\right)$ was sampled continuously at the mouth by an infrared carbon dioxide meter (Datex Normocap); arterial oxygen saturation $\left(\mathrm{SaO}_{2}\right)$ was monitored by an ear oximeter (Radiometer).

For mechanical studies an oesophageal latex balloon (length $10 \mathrm{~cm}$; air volume $0.5 \mathrm{ml}$ ) was introduced via the nose. A marker was placed on the polyethylene tubing exactly $45 \mathrm{~cm}$ from the balloon tip ${ }^{17}$ and adjustment began when this marker appeared at the external nares. The catheter was connected to a differential pressure transducer (Validyne). The maximal pleural pressure (Pplmax) was evaluated during the maximal sniff manoeuvre ${ }^{18}$ which was repeated until three measurements with less than $5 \%$ variability were recorded. The highest value of PPLmax obtained was used for subsequent analysis. Pleural pressure was also recorded during tidal breathing and PPL swings (PPLsw) were calculated as the difference between the pleural pressure measured at end expiration and end inspiration. PPL swings were expressed both as absolute values $\left(\mathrm{cm} \mathrm{H}_{2} \mathrm{O}\right)$ and as percentage of the maximal pleural pressure (PPLsw/Pplmax ratio), which represents the force required to breathe relative to the maximal inspiratory force available. Total lung resistance $(R L)$ was calculated by an isovolume method $^{19} ; \mathrm{RL} /$ PpLmax ratio was also calculated and represented the balance between the mechanical impediment to breathing and the ability of inspiratory muscles to develop force. The tension time index of the inspiratory muscles was calculated as the product of $\mathrm{TI} /$ Tтот $\times$ Pplsw/PrLmax. Mouth pressure during tidal breathing was measured using a pressure transducer (Statham P23ID).

The electromyographic activity (EMG) of the inspiratory muscles was recorded using a method previously described. ${ }^{2021}$ Electromyographic activity of parasternal muscles (EPS) was recorded from the second to third intercostal spaces parasternally and that of the diaphragm (EDI) from the lower anterolateral rib cage, from the sixth to seventh intercostal space on the midclavicular line, via large surface electrodes. Muscle action potentials ("raw") were differentially amplified, filtered between 100 and $1000 \mathrm{~Hz}$. The filtered EMG signal along with mouth pressure recording were displayed on a single beam storage oscilloscope (Tektronix 5115). EMG activity was full wave rectified and integrated over time (time constant $100 \mathrm{~ms}$ ) using a third order, low pass filter to provide a measurement of change in average electrical activity as a function of time, referred to as "moving time average". ${ }^{22-24}$ Inspiratory activity was quantified both as peak activity and as rate of rise (slope). The former was directly measured in arbitrary units and the latter obtained by dividing this value by the relevant inspiratory time. Because of the variability of the impedance between inspiratory muscles and electrodes, absolute values $(\mathrm{mV}$ ) are not comparable between different subjects. To overcome this problem and to obtain a reference value, EMG activity was measured whilst the subject performed an inspiratory capacity (IC) manoeuvre up to TLC connected to the pneumotachograph. ${ }^{25}$ This manoeuvre was repeated at least three times and in each subject both IC and the intensity of the recorded parasternal and diaphragmatic EMG was reproducible (less than $5 \%$ variability). The maximal level of this EMG activity was taken as a reference and the successive measurements have been expressed as a percentage of this value at TLC. As the inhalation of $\beta_{2}$ agonists could induce changes in lung volumes and in chest wall configuration, each patient performed TLC manoeuvres twice - first under control conditions and second after the inhalation of fenoterol. Thus, for each phase of the experiment EMG measurements were expressed as a percentage of the relevant value recorded at TLC. As EMG activity of an inspiratory muscle may include cardiac muscle activity, we checked cardiac artifacts to a manually gated ECG, when necessary, so that it would not contribute to the EMG.

The output of the carbon dioxide meter, flow signal, integrated flow signal, mouth pressure, oesophageal pressure, and the moving time average of both diaphragm and parasternal muscles were recorded continuously on a multichannel chart recorder (Gould TA4000).

\section{PROTOCOL}

On a pre-intervention day subjects underwent blood eosinophil count and baseline spirometric testing and the response to $400 \mu \mathrm{g}$ fenoterol was measured. Patients fulfilling the 
Table 1 Anthropometric characteristics, lung volumes, and arterial blood gas tensions of the nine patients with COPD

\begin{tabular}{|c|c|c|c|c|c|c|c|c|c|c|c|c|c|}
\hline \multirow[t]{2}{*}{ Subject } & \multirow{2}{*}{$\begin{array}{l}\text { Age } \\
\text { (years) }\end{array}$} & \multirow{2}{*}{$\begin{array}{l}\text { Height } \\
(\mathrm{cm})\end{array}$} & \multirow{2}{*}{$\begin{array}{l}\text { Weight } \\
(\mathrm{kg})\end{array}$} & \multicolumn{2}{|l|}{$F E V_{l}$} & \multicolumn{2}{|l|}{$V C$} & \multicolumn{2}{|l|}{$F R C$} & \multirow{2}{*}{$\begin{array}{l}R V \\
\text { (l) }\end{array}$} & \multirow{2}{*}{$\begin{array}{l}T L C \\
\text { (l) }\end{array}$} & \multirow{2}{*}{$\begin{array}{l}\mathrm{PaO}_{2} \\
(\mathrm{kPa})\end{array}$} & \multirow{2}{*}{$\begin{array}{l}\mathrm{PaCO}_{2} \\
(\mathrm{kPa})\end{array}$} \\
\hline & & & & $l$ & \%pred & $l$ & \%pred & $l$ & \%pred & & & & \\
\hline $\begin{array}{l}1 \\
2 \\
3 \\
4 \\
5 \\
6 \\
7 \\
8 \\
9\end{array}$ & $\begin{array}{l}61 \\
70 \\
69 \\
69 \\
67 \\
71 \\
63 \\
63 \\
58\end{array}$ & $\begin{array}{l}172 \\
170 \\
165 \\
169 \\
168 \\
162 \\
177 \\
163 \\
162\end{array}$ & $\begin{array}{l}72 \\
68 \\
84 \\
95 \\
45 \\
64 \\
89 \\
55 \\
63\end{array}$ & $\begin{array}{l}0.40 \\
0.73 \\
0.53 \\
0 \cdot 83 \\
0 \cdot 70 \\
0 \cdot 60 \\
0 \cdot 64 \\
0.43 \\
0.32\end{array}$ & $\begin{array}{l}12 \cdot 7 \\
26 \cdot 2 \\
20 \cdot 3 \\
29 \cdot 9 \\
25 \cdot 1 \\
24 \cdot 8 \\
19 \cdot 4 \\
16 \cdot 0 \\
11 \cdot 4\end{array}$ & $\begin{array}{l}3.87 \\
2.56 \\
2.51 \\
3 \cdot 25 \\
3.49 \\
4.04 \\
2 \cdot 19 \\
2 \cdot 22 \\
2 \cdot 36\end{array}$ & $\begin{array}{r}93 \cdot 6 \\
68 \cdot 1 \\
72 \cdot 1 \\
87 \cdot 2 \\
93 \cdot 8 \\
124 \cdot 5 \\
49 \cdot 9 \\
62.9 \\
65 \cdot 4\end{array}$ & $\begin{array}{l}5 \cdot 69 \\
4 \cdot 04 \\
4 \cdot 79 \\
5 \cdot 89 \\
5 \cdot 35 \\
5 \cdot 33 \\
6 \cdot 09 \\
4 \cdot 84 \\
6 \cdot 69\end{array}$ & $\begin{array}{l}163 \cdot 3 \\
114.8 \\
141 \cdot 2 \\
168 \cdot 2 \\
155 \cdot 3 \\
160 \cdot 0 \\
168 \cdot 3 \\
147 \cdot 9 \\
207.5\end{array}$ & $\begin{array}{l}4 \cdot 42 \\
3 \cdot 38 \\
4 \cdot 02 \\
3 \cdot 99 \\
4 \cdot 19 \\
3 \cdot 60 \\
5 \cdot 42 \\
3 \cdot 65 \\
5 \cdot 82\end{array}$ & $\begin{array}{l}8 \cdot 29 \\
5.94 \\
6 \cdot 52 \\
7 \cdot 24 \\
7 \cdot 68 \\
7 \cdot 65 \\
7 \cdot 61 \\
5 \cdot 86 \\
8 \cdot 19\end{array}$ & $\begin{array}{r}9 \cdot 18 \\
9 \cdot 59 \\
11 \cdot 33 \\
9 \cdot 99 \\
10 \cdot 55 \\
10 \cdot 11 \\
8 \cdot 22 \\
9 \cdot 48 \\
10 \cdot 23\end{array}$ & $\begin{array}{l}5 \cdot 85 \\
5.98 \\
5 \cdot 60 \\
5 \cdot 80 \\
5.98 \\
5 \cdot 76 \\
7 \cdot 65 \\
5 \cdot 71 \\
6 \cdot 69\end{array}$ \\
\hline $\begin{array}{l}\text { Mean } \\
\text { SE }\end{array}$ & $\begin{array}{c}65 \cdot 7 \\
1.51\end{array}$ & $\begin{array}{c}168 \\
1 \cdot 69\end{array}$ & $\begin{array}{c}70 \cdot 3 \\
5 \cdot 28\end{array}$ & $\begin{array}{l}0.57 \\
0.06\end{array}$ & $\begin{array}{c}20 \cdot 6 \\
2 \cdot 12\end{array}$ & $\begin{array}{l}2 \cdot 9 \\
0 \cdot 24\end{array}$ & $\begin{array}{c}79 \cdot 7 \\
7 \cdot 47\end{array}$ & $\begin{array}{l}5 \cdot 4 \\
0 \cdot 26\end{array}$ & $\begin{array}{r}158.5 \\
8.3\end{array}$ & $\begin{array}{l}4 \cdot 3 \\
0 \cdot 28\end{array}$ & $\begin{array}{l}7 \cdot 2 \\
0 \cdot 30\end{array}$ & $\begin{array}{l}9 \cdot 85 \\
0 \cdot 29\end{array}$ & $\begin{array}{l}6 \cdot 11 \\
0 \cdot 22\end{array}$ \\
\hline
\end{tabular}

$\mathrm{FEV}_{1}=$ forced expiratory volume in one second; VC = vital capacity; FRC = functional residual capacity; $\mathrm{RV}=$ residual volume; $\mathrm{TLC}=$ total lung capacity; $\mathrm{PaO}{ }_{2}=$ arterial partial pressure of oxygen; $\mathrm{PaCO}_{2}=$ arterial partial pressure of carbon dioxide.

entry criteria were enrolled. On the study day $\mathrm{FEV}_{1}, \mathrm{VC}, \mathrm{FRC}$, and PPLmax were measured. Patients then breathed quietly through the pneumotachograph and, after a 10 minute acclimatisation period, PPLsw, breathing pattern, and electromyographic activity of inspiratory muscles were recorded. On completion of baseline measurements each patient inhaled $800 \mu \mathrm{g}$ fenoterol from a metered dose inhaler via a space device. Twenty and 40 minutes after inhalation we recorded PPLsw, breathing pattern, EPS and EDI during quiet breathing and, finally, the $\mathrm{FEV}_{1}$. After completion of the 40 minutes recording, VC and FRC were remeasured. In five patients we also studied the effects of placebo administration (single blind design) following the same experimental protocol.

\section{DATA ANALYSIS}

Mean values and standard errors of mean have been calculated for all variables. Data obtained in control conditions and at 20 and 40 minutes after inhalation of fenoterol were compared by two way analysis of variance. Subsequent comparisons were performed by the Bonferroni test. Changes in VC and FRC were analysed by the Student's $t$ test for paired samples. All statistical analyses were carried out using the Statgraphics 6.0 package (Manugistics Inc, Rockville, Maryland, USA).

\section{Results}

Anthropometric details, lung volumes, and arterial blood gas tensions are summarised in table 1. Patients exhibited severe airway obstruction $\left(<40 \% \mathrm{FEV}_{1}\right)$, moderate hyperinflation (FRC), and a reduction of VC. $\mathrm{PaO}_{2}$ was slightly reduced and patients nos 7 and 9 were hypercapnic. After inhalation of $800 \mu \mathrm{g}$ fenoterol $\mathrm{FEV}_{1}$ increased significantly and FRC decreased significantly; changes in VC were not significant (table 2 ).

\section{BREATHING PATTERN MEASUREMENTS}

Mean breathing pattern, minute ventilation, and $\mathrm{PeTCO}_{2}$ under control conditions and 20 and 40 minutes after inhalation of fenoterol are shown in fig 1. After fenoterol $\mathrm{T}_{\mathrm{I}}$ and $\mathrm{TE}$ lengthened, decreasing respiratory frequency (from $17.6(1.7)$ to $14.6(1.4)$ breaths $/ \mathrm{min}$ under control conditions and at 40 minutes respectively, $\mathrm{p}<0.005)$, and $\mathrm{VT}$ increased $(p<0.01)$. VE did not change, and VT/TI exhibited a small but significant decrease $(p<0.05)$ (fig 1). No significant change was observed in $\mathrm{TI} / \mathrm{T}$ тот. Following the increase in VT, PETCO $_{2}$ decreased significantly (fig 1) but arterial oxygen saturation did not change (from $92.5(0 \cdot 81) \%$ to $92 \cdot 2(0 \cdot 70) \%)$. A small but significant increase in heart rate was noted (from $84.1(5.6)$ to $90.6(6.4)$ beats $/ \mathrm{min}$, $\mathrm{p}<0.01)$ without any arrhythmias.

\section{INSPIRATORY MUSCLE STRENGTH MEASUREMENTS}

The patients had reduced inspiratory muscle strength with a low value of PpLmax, and a small but significant increase was observed both

Table 2 Mean (SE) lung volumes of the patients with COPD before and after inhalation of $800 \mu \mathrm{g}$ fenoterol (nine patients) and of placebo (five patients)

\begin{tabular}{|c|c|c|c|c|c|c|}
\hline & \multicolumn{2}{|l|}{$F E V_{1}$} & \multicolumn{2}{|l|}{$V C$} & \multicolumn{2}{|l|}{$F R C$} \\
\hline & $l$ & \%pred & $l$ & \%pred & $l$ & \%pred \\
\hline Before fenoterol & $\begin{array}{c}0.57 \\
(0.06)\end{array}$ & $\begin{array}{l}20 \cdot 6 \\
(2 \cdot 12)\end{array}$ & $\begin{array}{l}2 \cdot 9 \\
(0 \cdot 24)\end{array}$ & $\begin{array}{l}79 \cdot 7 \\
(7 \cdot 47)\end{array}$ & $\begin{array}{c}5 \cdot 41 \\
(0 \cdot 26)\end{array}$ & $\begin{array}{r}158 \cdot 5 \\
(8 \cdot 29)\end{array}$ \\
\hline After fenoterol & 0.71 & $25 \cdot 7$ & $3 \cdot 1$ & $84 \cdot 3$ & $5 \cdot 17$ & $152 \cdot 1$ \\
\hline & $(0.04)$ & $(1.77)$ & $(0 \cdot 27)$ & $(9 \cdot 20)$ & $(0 \cdot 33)$ & $(10 \cdot 14)$ \\
\hline$t$ & 5.64 & 5.44 & 1.49 & 1.45 & $4 \cdot 00$ & 3.70 \\
\hline $\mathrm{p}$ & $<0.0005$ & $<0.0005$ & NS & NS & $<0.01$ & $<0.01$ \\
\hline Before placebo & $\begin{array}{c}0.54 \\
(0.09)\end{array}$ & $\begin{array}{l}18 \cdot 38 \\
(2.52)\end{array}$ & $\begin{array}{c}2 \cdot 8 \\
(0 \cdot 23)\end{array}$ & $\begin{array}{l}72 \cdot 1 \\
(3 \cdot 48)\end{array}$ & $\begin{array}{c}5 \cdot 33 \\
(0 \cdot 38)\end{array}$ & $\begin{array}{l}158 \cdot 7 \\
(12 \cdot 8)\end{array}$ \\
\hline After placebo & $\begin{array}{c}0.6 \\
(0.10)\end{array}$ & $\begin{array}{l}20 \cdot 34 \\
(2.72)\end{array}$ & $\begin{array}{c}3.0 \\
(0.33)\end{array}$ & $\begin{array}{l}78.64 \\
(5.45)\end{array}$ & $\begin{array}{c}5 \cdot 10 \\
(0.43)\end{array}$ & $\begin{array}{l}151 \cdot 7 \\
(14.71)\end{array}$ \\
\hline$t$ & $\begin{array}{l}(0.10) \\
1.18\end{array}$ & $\begin{array}{c}(2 \cdot 72) \\
1.05\end{array}$ & $\begin{array}{c}(0.33) \\
1.87\end{array}$ & $\begin{array}{l}(5.45) \\
1.81\end{array}$ & $\begin{array}{c}(0.43) \\
1.78\end{array}$ & $\begin{array}{c}(14.71) \\
1.78\end{array}$ \\
\hline $\mathrm{p}$ & NS & NS & NS & NS & NS & NS \\
\hline
\end{tabular}

For abbreviations see footnote to table 1 . 

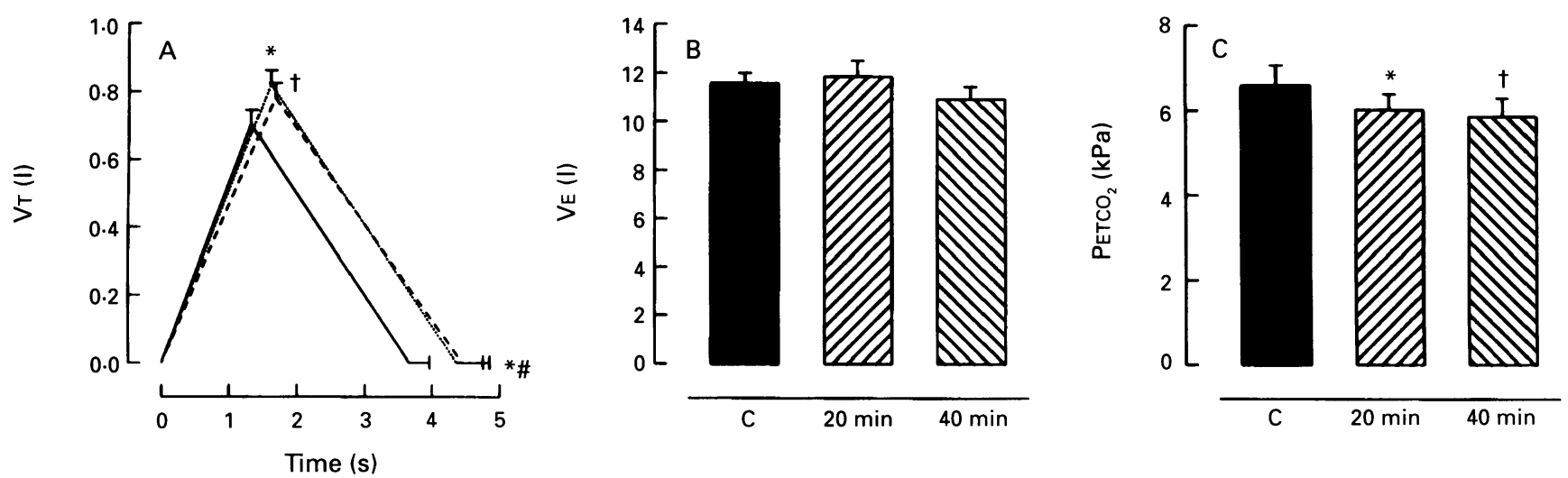

Figure 1 (A) Schematic representation of breathing pattern under control conditions (continuous line), and 20 (dotted line) and 40 (broken line) minutes after the inhalation of $800 \mu \mathrm{g}$ fenoterol. $(B)$ and (C) Minute ventilation (VE) and end tidal PCO, under control conditions (C), and 20 and 40 minutes after the inhalation of $800 \mu \mathrm{g}$ fenoterol, respectively. ${ }^{*} C$ versus 20 min, $p<0 \cdot 05 ;+C$ versus 40 min, $p<0 \cdot 05$. VT=tidal volume, VE $=$ minute ventilation, $\mathrm{PETCO}_{2}=$ end tidal partial pressure of carbon dioxide.

20 and 40 minutes after fenoterol inhalation $(\mathrm{p}<0 \cdot 05)$ (fig 2).

\section{MECHANICAL MEASUREMENTS}

Swings of pleural pressure during tidal breathing, expressed either in absolute values or as percentage PrLmax, were increased under control conditions and this value fell significantly after fenoterol (table 3 and fig 2). All patients had raised values of $R_{L}$ and $R_{L} /$ PpLmax ratio; both variables exhibited significant reductions after fenoterol (table 3). Tension time index (TTI) of inspiratory muscles decreased significantly after fenoterol (table 3); since no variation in $\mathrm{TI} / \mathrm{TTOT}$ was observed after fenoterol, the change in TTI appeared to be due mainly to a decrease in the Pplsw/Prlmax ratio.

\section{ELECTROMYOGRAPHIC MEASUREMENTS}

Under control conditions patients showed high levels of both EDI and EPS, expressed either as peak or slope (normal values of our laboratory for age-matched and sex-matched subjects are: EDI $4 \cdot 1 \%$ TLC, EDI/TI $1.93 \% \mathrm{TLC} / \mathrm{s}$; EPS $3.43 \%$ TLC, and EPS/Ti $1.85 \%$ TLC/s). With fenoterol a marked and significant reduction in both peak and slope were observed (table 3).

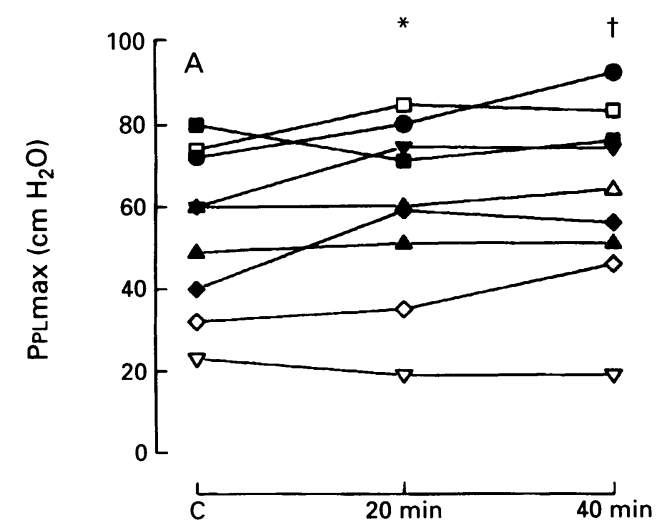

RELATIONSHIPS BETWEEN CHANGES IN VARIABLES

For each variable, values recorded at 20 and 40 minutes after fenoterol inhalation were similar. We therefore plotted the different variables in terms of changes recorded between control and 40 minutes after fenoterol. The results of these plots are summarised in table 4 . In particular, changes in VT and $\mathrm{R} f$ were significantly related to changes in both PPLsw (\% PPLmax) and the $\mathrm{RL} /$ Pplmax ratio (table 4 and fig 3 ) but not to changes in $R_{L}$ or $\mathrm{FEV}_{1}$; similarly, changes in EDI/TI and EPS/TI were related to changes in Pplsw (\% Pplmax) and Ru/Pplmax ratio (table 4). Finally, changes in Pplmax did not relate to changes in FRC.

\section{PLACEBO EFFECTS}

With placebo no significant change was observed in the studied variables (tables 2 and 3 ). Only heart rate exhibited small but significant changes (from 80.6 to 84.1 and 82.9 under control conditions and at 20 and 40 minutes after fenoterol inhalation, respectively).

\section{Discussion}

Our results suggest that patients with COPD and severe obstruction of the airways have reduced inspiratory muscle strength, a rapid and shallow pattern of breathing, high pleural

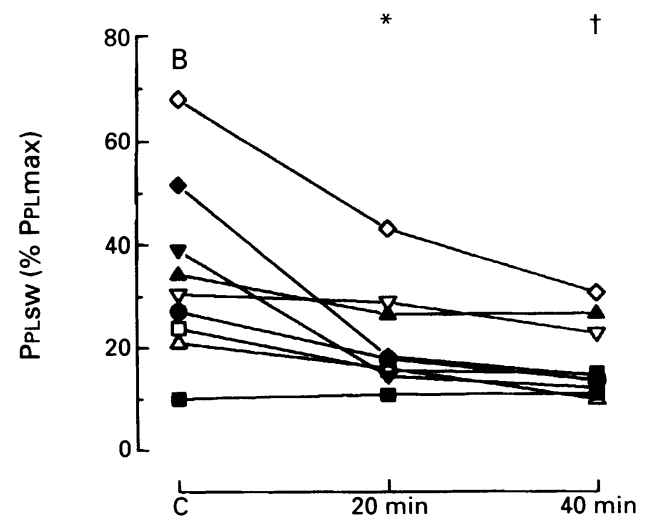

Figure 2 (A) Maximal pleural pressure (PPLmax) during sniff manoeuvre and (B) pleural pressure swings (PPLSW) expressed as \% of PPLmax during tidal breathing under control conditions $(C)$ and 20 and 40 minutes after the inhalation of $800 \mu \mathrm{g}$ fenoterol. ${ }^{*} C$ versus 20 min, $p<0.05 ;+C$ versus 40 min, $p<0.05$. 

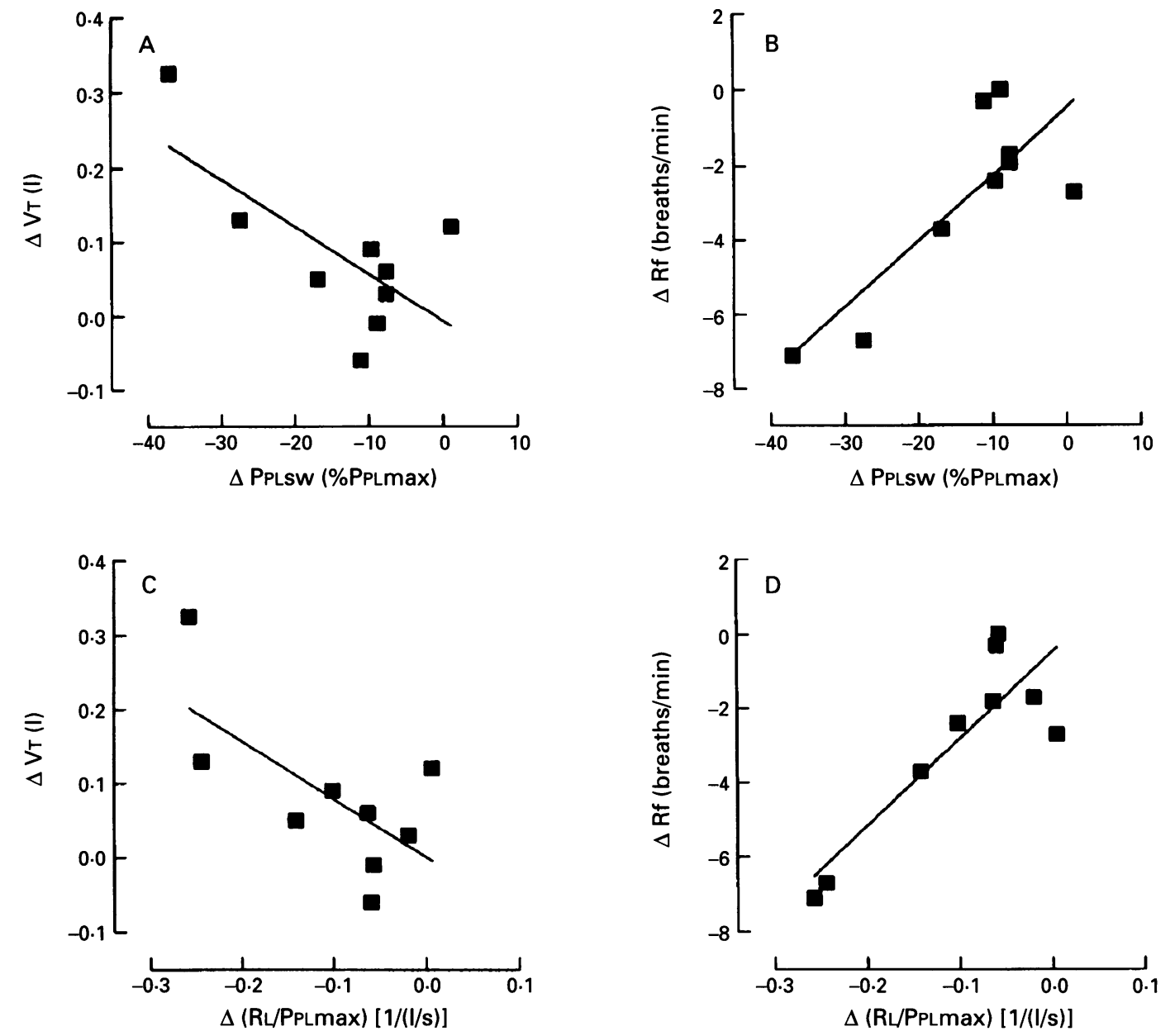

Figure 3 Relationships between changes in tidal volume $(V T)$ and respiratory frequency $(R f)$ with $(A, B)$ changes in PPLSW (\% PPLmax) and (C,D) RL/PPLmax. Abbreviations as in table 3. For explanation see text.

pressure swings during tidal breathing, and high activity of inspiratory muscles as assessed by electromyographic activity of both parasternal and diaphragm muscles. Airflow obstruction plays an important part in determining these alterations, as significant changes were measured after inhalation of a large dose of fenoterol.

Decreased inspiratory muscle strength is common in patients with COPD, particularly when there is accompanying hyperinflation. The reduction in maximal inspiratory pressure

Table 3 Mean (SE) pleural pressure swings, total lung resistance, electromyographic activity of diaphragm and parasternal muscles under control conditions, and 20 and 40 minutes after the inhalation of fenoterol (nine patients) and placebo (five patients)

\begin{tabular}{|c|c|c|c|c|c|c|c|c|}
\hline & $\begin{array}{l}\text { PPLsw } \\
\left(\mathrm{cm} \mathrm{H} \mathrm{H}_{2} \mathrm{O}\right)\end{array}$ & $\begin{array}{l}R L \\
\left(\mathrm{~cm} \mathrm{H}_{2} \mathrm{O}\right) /(\mathrm{l} / \mathrm{s})\end{array}$ & $\begin{array}{l}R L / P P L \max \\
1(l / s)\end{array}$ & $T T_{I}$ & $\begin{array}{l}E D I \\
(\% T L C)\end{array}$ & $\begin{array}{l}E D I / T I \\
(\% T L C / s)\end{array}$ & $\begin{array}{l}\text { EPS } \\
(\% T L C)\end{array}$ & $\begin{array}{l}E P S / T_{I} \\
(\% T L C / s)\end{array}$ \\
\hline \multicolumn{9}{|l|}{ Fenoterol $(n=9)$} \\
\hline Control & $\begin{array}{l}16.42 \\
(1.99)\end{array}$ & $\begin{array}{l}12 \cdot 85 \\
(0.99)\end{array}$ & $\begin{array}{c}0 \cdot 27 \\
(0.04)\end{array}$ & $\begin{array}{c}0 \cdot 121 \\
(0.020)\end{array}$ & $\begin{array}{c}29 \cdot 5 \\
(5 \cdot 8)\end{array}$ & $\begin{array}{c}26 \cdot 8 \\
(6 \cdot 3)\end{array}$ & $\begin{array}{c}31 \cdot 1 \\
(7 \cdot 9)\end{array}$ & $\begin{array}{c}27 \cdot 3 \\
(7 \cdot 6)\end{array}$ \\
\hline $20 \mathrm{~min}$ & $\begin{array}{l}11.19 \\
(1.06)\end{array}$ & $\begin{array}{c}9.04 \\
(0.43)\end{array}$ & $\begin{array}{c}0.18 \\
(0.09)\end{array}$ & $\begin{array}{c}0.080 \\
(0.013)\end{array}$ & $\begin{array}{l}18 \cdot 7 \\
(4 \cdot 1)\end{array}$ & $\begin{array}{l}13 \cdot 5 \\
(3 \cdot 3)\end{array}$ & $\begin{array}{l}19.0 \\
(2.9)\end{array}$ & $\begin{array}{l}13 \cdot 3 \\
(2 \cdot 3)\end{array}$ \\
\hline $40 \mathrm{~min}$ & $\begin{array}{l}9.95 \\
(1 \cdot 13)\end{array}$ & $\begin{array}{c}8.85 \\
(0.39)\end{array}$ & $\begin{array}{c}0.167 \\
(0.03)\end{array}$ & $\begin{array}{c}0.066 \\
(0.029)\end{array}$ & $\begin{array}{c}14.9 \\
(3 \cdot 8)\end{array}$ & $\begin{array}{l}9 \cdot 9 \\
(2 \cdot 7)\end{array}$ & $\begin{array}{l}16 \cdot 6 \\
(2 \cdot 1)\end{array}$ & $\begin{array}{c}10.9 \\
(1.8)\end{array}$ \\
\hline \multicolumn{9}{|l|}{ Analysis of variance } \\
\hline$F$ & $16 \cdot 01$ & $21 \cdot 156$ & $10 \cdot 44$ & $12 \cdot 356$ & $9 \cdot 84$ & $9 \cdot 04$ & $4 \cdot 29$ & $4 \cdot 80$ \\
\hline p & $<0.001$ & $<0.001$ & $<0.005$ & $<0.001$ & $<0.005$ & $<0.005$ & $<0.05$ & $<0.05$ \\
\hline \multicolumn{9}{|l|}{ Bonferroni test } \\
\hline $\mathrm{p}\left(\mathrm{C} v 20^{\prime}\right)$ & $<0.05$ & $<0.05$ & $<0.05$ & $<0.05$ & $<0.05$ & $<0.05$ & NS & NS \\
\hline $\mathrm{p}\left(\mathrm{C} v 40^{\prime}\right)$ & $<0.05$ & $<0.05$ & $<0.05$ & $<0.05$ & $<0.05$ & $<0.05$ & $<0.05$ & $<0.05$ \\
\hline $\mathrm{p}\left(20^{\prime} v 40^{\prime}\right)$ & NS & NS & NS & NS & NS & NS & & \\
\hline \multicolumn{9}{|l|}{ Placebo $(n=5)$} \\
\hline Control & $\begin{array}{c}16.52 \\
(1.97)\end{array}$ & $\begin{array}{c}14 \cdot 07 \\
(1 \cdot 21)\end{array}$ & $\begin{array}{c}0.29 \\
(0.06)\end{array}$ & $\begin{array}{c}0.120 \\
(0.024)\end{array}$ & $\begin{array}{c}28.98 \\
(3.0)\end{array}$ & $\begin{array}{c}25 \cdot 37 \\
(4 \cdot 9)\end{array}$ & $\begin{array}{l}29 \cdot 18 \\
(5 \cdot 88)\end{array}$ & $\begin{array}{l}24 \cdot 45 \\
(3 \cdot 63)\end{array}$ \\
\hline $20 \mathrm{~min}$ & $17 \cdot 4$ & 14.00 & $0 \cdot 29$ & $0 \cdot 115$ & $29 \cdot 86$ & $27 \cdot 37$ & 31.67 & 28.79 \\
\hline & $(1 \cdot 77)$ & $(1 \cdot 14)$ & $(0 \cdot 06)$ & $(0 \cdot 020)$ & $(2 \cdot 3)$ & (3.7) & $(6 \cdot 7)$ & $(5 \cdot 6)$ \\
\hline $40 \mathrm{~min}$ & $18 \cdot 3$ & $14 \cdot 26$ & $0 \cdot 30$ & $0 \cdot 126$ & $29 \cdot 44$ & $27 \cdot 30$ & $31 \cdot 28$ & $27 \cdot 80$ \\
\hline \multicolumn{9}{|l|}{ Analysis of variance } \\
\hline$F$ & 1.555 & 0.018 & 0.006 & $0 \cdot 817$ & 0.065 & $0 \cdot 188$ & 0.542 & 0.977 \\
\hline $\mathrm{p}$ & NS & NS & NS & NS & NS & NS & NS & NS \\
\hline
\end{tabular}

PPLSW = swings of pleural pressure during tidal breathing; $\mathrm{RL}=$ total lung resistance; PPLmax = maximal pleural pressure during maximal sniff manoeuvre; TTI tension time index of inspiratory muscles calculated as the product of TI/TTOT $\times$ PPLsw/PPLmax; EDI = peak of electromyographic activity of diaphragm; EDI/TI $=$ rate of rise of electromyographic activity of diaphragm; EPS = peak of electromyographic activity of parasternal muscles; EPS/TI= rate of rise of electromyographic activity of parasternal muscles. 
Table 4 Regression equations between changes in studied variables

\begin{tabular}{|c|c|c|c|c|}
\hline Independent variable & Dependent variable & Regression equation & $\begin{array}{l}\text { Correlation } \\
\text { coefficient (r) }\end{array}$ & $p$ \\
\hline 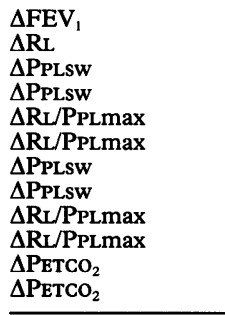 & $\begin{array}{l}\Delta \text { PPLSW } \\
\Delta \text { PPLSW } \\
\Delta \mathrm{VT} \\
\Delta \mathrm{Rf} \\
\Delta \mathrm{VT} \\
\Delta \mathrm{Rf} \\
\Delta \mathrm{EDI} / \mathrm{TI} \\
\Delta \mathrm{EPS} / \mathrm{TI} \\
\Delta \mathrm{EDI} / \mathrm{TI} \\
\Delta \mathrm{EPS} / \mathrm{TI} \\
\Delta \mathrm{EDI} / \mathrm{TI} \\
\Delta \mathrm{EPS} / \mathrm{TI}\end{array}$ & $\begin{array}{l}y=0 \cdot 65-51 \cdot 20 x \\
y=-0 \cdot 14+1 \cdot 6 x \\
y=-0 \cdot 006-0 \cdot 006 x \\
y=-0 \cdot 47+0 \cdot 18 x \\
y=0 \cdot 0002-0 \cdot 78 x \\
y=-0 \cdot 51-23 \cdot 3 x \\
y=0 \cdot 87+1 \cdot 28 x \\
y=6 \cdot 96+1 \cdot 68 x \\
y=0 \cdot 75+169 x \\
y=4 \cdot 04+196 x \\
y=-3 \cdot 54+3 \cdot 32 x \\
y=2 \cdot 26+4 \cdot 65 x\end{array}$ & $\begin{array}{r}-0.83 \\
0.95 \\
-0.68 \\
0 \cdot 82 \\
-0 \cdot 67 \\
0.86 \\
0.91 \\
0.90 \\
0.96 \\
0 \cdot 84 \\
0 \cdot 81 \\
0 \cdot 86\end{array}$ & $\begin{array}{l}<0.01 \\
<0.002 \\
<0.05 \\
<0.01 \\
<0.05 \\
<0.005 \\
<0.001 \\
<0.001 \\
<0.0001 \\
<0.005 \\
<0.01 \\
<0.005\end{array}$ \\
\hline
\end{tabular}

$\mathrm{VT}=$ tidal volume; $\mathrm{R} f=$ respiratory frequency; $\mathrm{PETCO}_{2}=$ end tidal partial pressure of carbon dioxide. For other abbreviations see footnotes to tables 1 and 3 .

is mainly dependent on increased lung volume, which places the inspiratory muscles at a mechanical disadvantage. ${ }^{42627}$ However, an increase in the maximal strength of the inspiratory muscles following a $\beta_{2}$ agonist has been reported, ${ }^{28-30}$ with an increase in inspiratory muscle fibre length following a fall in end expiratory lung volume ${ }^{2930}$; a direct effect of $\beta_{2}$ agonists on the respiratory muscles ${ }^{28}$ may also have a role. In the present study fenoterol caused a small but significant increase in PPLmax and a decrease in FRC, but no significant relationship between these changes was found. The present data confirm the ability of $\beta_{2}$ agonists to increase inspiratory muscle strength, but the small changes cast doubt on the clinical significance of these increases.

Change in pleural pressure during tidal breathing - that is, PPL swing - is an expression of the mechanical load that inspiratory muscles have to sustain to maintain ventilation, as shown by the close relationship between changes in PPLsw and RL or FEV 1 with fenoterol. The balance between the mechanical impediment to breathing and the ability of inspiratory muscles to develop force - that is, the ratio of RI or PPLSw to PPLmax - reflects the relative force required for inspiration. ${ }^{8}$ The high values of Pplsw/Pplmax and RL/PPLmax we obtained indicate that at each breath patients had to use a high proportion of their maximal strength in order to achieve airflow. Fenoterol caused a dramatic decrease in both RL and PPLsw and a small increase in PPLmax; the consequent decrease in $R_{L} / P_{P L m a x}$ indicated a decrease in inspiratory muscle loading relative to the maximal available force. The high TTI values in control conditions and their significant decrease after fenoterol confirm this interpretation.

Fenoterol induced small but significant changes in the breathing pattern (increase in $\mathrm{VT}$ and reduction in $\mathrm{R} f$ ). In order to achieve the increased mechanical load a higher pressure must be developed with each breath and, in the presence of the reduced inspiratory muscle strength (increased RL/PpLmax ratio), a high proportion of the available force will be used at each breath (high Pplsw/Pplmax ratio). In these conditions central respiratory activity is directed towards a shallower pattern of breathing and an increase in respiratory frequency, the latter offsetting the reduction in VT and allowing VE to remain in the normal range. ${ }^{8}$ Fenoterol induced significant changes in both
$\mathrm{VT}$ (increase) and $\mathrm{R} f$ (decrease), which related to changes in PPLsw (\%PPLmax) and $\mathrm{RL} /$ Pplmax ratio but not to changes in either RL or $\mathrm{FEV}_{1}$. Consistent with the hypothesis of Rochester, ${ }^{8}$ these findings indicate that it is the ratio between the mechanical load and the available strength rather than the mechanical load itself which determines the pattern of breathing.

Patients with COPD have a high neural inspiratory drive compared with normal subjects. ${ }^{1-4}$ Our patients showed high values of EMG activity of both diaphragm and intercostal muscles and this suggests that a high neural inspiratory drive was present. This conclusion may be correct if one considers the EMG activity of the inspiratory muscles to be a reliable index of neural inspiratory drive. We have criticised the use of either surface or oesophageal EMG recordings for assessing neural drive in humans. ${ }^{42031}$ However, a close correlation between changes in electrical activity of the phrenic nerve and the diaphragm has been reported in dogs, during both normal breathing and obstructed breathing, ${ }^{32}$ and data are available to support the contention that the slope of the "moving time average" is a reliable measure for assessing neural inspiratory drive to the respiratory muscles both in normal and in disease states. ${ }^{420212431}$ The close relationship between EMG and PpLsw further supports the hypothesis that surface EMG of inspiratory muscles may be a useful tool for evaluating inspiratory muscle activation for clinical purposes. Both mechanical (pulmonary and chest wall $^{167}$ and chemical afferents ${ }^{19}$ may be involved in the increased neural inspiratory drive observed in our patients. Fenoterol induced a marked decrease in EDI and EPs in terms of both peak (49.5\% and $46.62 \%$, respectively) and slope $(63.05 \%$ and $60.02 \%$, respectively) activity. The close relationship between decrease in either PPLSw or RL/PPLmax ratio and decrease in EMG activity of both diaphragm and parasternal muscles (table 4) supports the hypothesis that mechanical impairment has an important role in determining the increase in inspiratory neural drive. Chemical factors may also play a part, as shown by the significant relationship between changes in PETCO $_{2}$ and changes in EDI/TI and EPS/TI.

In conclusion, in patients with severe COPD high doses of fenoterol induce a significant decrease in total lung resistance and a concomitant decrease in PpLsw and in PpLsw/ 
Pplmax. Decrease in inspiratory muscle loading relative to the maximal available strength, as expressed by the RL/PPLmax and PPLsw/ Pplmax ratios, appears to be the major determinant of both changes in breathing pattern and decrease in inspiratory muscle activation. These findings may be of clinical relevance since $\beta_{2}$ agonists at high doses may induce a significant improvement in respiratory mechanics with minor cardiac side effects.

This study was supported by grants from the Ministero dell' Università e della Ricerca Scientifica e Tecnologica of Italy.

1 Sorli J, Grassino A, Lorange G, Milic-Emili J. Control of breathing in patients with chronic obstructive pulmonary disease. Clin Sci Mol Med 1978;54:295-305.

2 Gribbin HR, Gardiner IT, Heinz III GJ, Gibson GJ, Pride NB. Role of impaired inspiratory muscle function in limiting the ventilatory response to carbon dioxide in chronic airflow obstruction. Clin Sci 1983;64:487-95.

3 Scano G, Gigliotti F, Duranti R, Spinelli A, Gorini M, Schiavina $M$. Changes in ventilatory muscle function with negative pressure ventilation in patients with severe negative pressure ventilation
COPD. Chest 1990;97:322-7.

4 Gorini M, Spinelli A, Ginanni R, Duranti R, Gigliotti F Scano G. Neural respiratory drive and neuromuscular coupling in patients with chronic obstructive pulmonary disease (COPD). Chest 1990;98:1179-86.

5 Cohen CA, Gary Zagelbaum CM, Roussos C, Macklem PT. Clinical manifestations of inspiratory muscle fatigue. Am $\mathcal{F}$ Med 1982;73:308-16.

6 Roussos C, Moxham J. Respiratory muscle fatigue. In: Roussos C, Macklem PT, eds. The thorax (part B). New York: Marcel Dekker, 1985:829-70.

7 Altose MD, McCauley WC, Kelsen SG, Cherniak NS Effects of hypercapnia and inspiratory flow-resistive loading on respiratory activity in chronic airway obstruction. ing on respiratory activity in

8 Rochester DF. Respiratory muscle weakness, pattern of breathing, and $\mathrm{CO}_{2}$ retention in chronic obstructive pulmonary disease. Am Rev Respir Dis 1991;143:901-3.

Lee KD, Bishop JM. The reflex hypoxic drive in patient with chronic bronchitis. Clin Sci Mol Med 1974;46:347-56.

10 Mann J, Bradley CA, Anthonisen NR. Occlusion pressure in acute bronchospasm induced by methylcholine. Respir Physiol 1978;33:339-47.

11 Kelsen SG, Prestel TF, Cherniak NS, Chester EH, Chandle Deal Jr E. Comparison of the respiratory responses to external resistive loading and bronchoconstriction. $\mathcal{f}$ Clin Invest 1981;67:1761-8.

12 Gorini M, Spinelli A, Gigliotti F, Duranti R, Arcangeli P, Scano G. Changes in neural drive (EMGd) and neuromuscular coupling during histamine-induced bronchomuscular coupling during histamine-induced bronchoconstrictio.

13 Oliven A, Cherniak NS, Chandler Deal E, Kelsen SG. The effects of acute bronchoconstriction on respiratory activity in patients with chronic obstructive pulmonary disease. Am Rev Respir Dis 1985;131:236-41.
14 Pardy RL, Rivington RN, Milic-Emili J, Mortola JP. Control of breathing in chronic obstructive pulmonary disease. The effect of histamine inhalation. Am Rev Respir Dis 1982;125:6-11.

15 American Thoracic Society. Chronic bronchitis, asthma and pulmonary emphysema. A statement by the committee on diagnostic standards for non turberculous respiratory disease. Am Rev Respir Dis 1962;85:762-8.

16 European Community for Coal and Steel. Standardization of lung function test. Bull Eur Physiopathol Respir 1983 19:1-95.

17 Milic-Emili J, Mead J, Turner JM, Glauser EM. Improved technique for estimating pleural pressure from esophageal balloons. F Appl Physiol 1964;19:207-11.

18 Miller MJ, Moxham J, Green M. The maximal sniff in the assessment of diaphragm function in man. Clin Sci 1985 ; 69:91-6.

19 Frank NR, Mead J, Ferris BG Jr. The mechanical behaviour of the lungs in healthy elderly persons. $\mathcal{F}$ Clin Invest 1957 36:1680-7.

20 Spinelli A, Marconi G, Gorini M, Pizzi A, Scano G. Control of breathing in patients with myasthenia gravis. Am Rev Respir Dis 1992;145:1359-66.

21 Duranti R, Gheri RG, Gorini M, Gigliotti F, Spinelli A Fanelli A, et al. Control of breathing in patients with severe hypothyroidism. Am f Med 1993;95:29-37.

22 Evanich MJ, Lopata M, Lourenco RV Analytical methods for the study of electrical activity in respiratory nerves and muscles. Chest 1976;70(Suppl): 158-62.

23 Evanich MJ, Bruce E, Eldridge FL Goldman M, Lopata M, Lourenço RV, et al. Conference report: Workshop on assessment of respiratory control in humans: IV. Measurement of the electrical activity in respiratory muscles. $\mathrm{Am}$ Rev Respir Dis 1977;115:541-8.

24 Lopata M, Evanich MJ, Lourenço RV. Quantification of diaphragmatic EMG response to $\mathrm{CO}_{2}$ rebreathing in humans. F Appl Physiol 1977;43:262-70.

25 Grassino A, Goldman MD, Mead J, Sears TA. Mechanics of the human diaphragm during voluntary contraction statics. 7 Appl Physiol: Respir Environ Exerc Physiol 1978; 44:829-39.

26 Bégin $P$, Grassino A. Inspiratory muscle dysfunction and chronic hypercapnia in chronic obstructive pulmonary chronic hypercapnia in chronic obstructive

27 Rochester DF, Braun NMT. Determinants of maximal inspiratory pressure in chronic obstructive pulmonary disease. Am Rev Respir Dis 1985;132:42-7.

28 Derom E, Janssens S, Gurrieri G, Tjandramaga TB, Decramer M. Effects of broxaterol and theophylline on fatigued canine diaphragm in vivo. Am Rev Respir Dis 1992 146:22-5.

29 Dal Vecchio L, Polese G, Poggi R, Rossi A. "Intrinsic" positive end-expiratory pressure in stable patients with chronic obstructive pulmonary disease. Eur Respir 71990 3:74-80.

30 Gigliotti F, Gurrieri G, Duranti R, Gorini M, Scano G. Effects of intravenous broxaterol on respiratory drive and neuromuscular coupling in COPD patients. Eur Respir $f$ neuromuscular

31 Gorini M, Ginanni R, Spinelli A, Duranti R, Andreotti L, Scano $\mathrm{G}$. Inspiratory muscle strength and respiratory drive in patients with rheumatoid arthritis. Am Rev Respir Dis 1990;142:289-94.

32 Lourenço RV, Cherniack NS, Malm JR, Fishman AP. Nervous output from the respiratory center during obstructed breathing. f Appl Physiol 1966;21:527-33. 